\title{
Propuesta para el uso de TDICs en comunidades educativas rurales. Una experiencia en escuelas albergue
}

\author{
Sergio Rocabado ${ }^{1,3}$, Emilce Ottavianelli ${ }^{1,2}$, Carlos Cadena ${ }^{1,3}$ \\ ${ }^{1}$ Consejo de Investigación de la Universidad Nacional de Salta (CIUNSa) \\ Av. Bolivia 5150 - Salta - Argentina \\ ${ }^{1}$ Instituto de Investigación para la Industria Química (INIQUI) \\ Av. Bolivia 5150 - Salta - Argentina \\ ${ }^{3}$ Instituto de Investigaciones en Energía No Convencional (INENCO) \\ Av. Bolivia 5150 - Salta - Argentina \\ srocabado@di.unsa.edu.ar, ottavianelli@exa.unsa.edu.ar , \\ cadenacinenco@gmail.com
}

\begin{abstract}
Resumen. Este trabajo presenta una contribución para el uso de TDICs en comunidades educativas rurales ubicadas en zonas aisladas que se encuentran fuera del alcance de los centros de distribución de energía. Se propone un escenario de trabajo que posibilita a maestros y alumnos el acceso a Internet mediante el uso de celulares y tecnologías de comunicación de bajo consumo energético, para abastecer de energía a los dispositivos móviles se desarrolló un sistema fotovoltaico portátil inteligente. Utilizando el escenario propuesto se realizó una experiencia en una escuela albergue de la provincia de Salta, los resultados alcanzados fueron positivos y altamente significativos desde el punto de vista educativo.
\end{abstract}

\begin{abstract}
This paper presents a contribution to the use of TDICs in rural educational communities located in isolated areas that are beyond the reach of energy distribution centres. We propose a scenario that enables teachers and students to access the Internet through the use of cell phones and communication technologies with low energy consumption. A smart portable photovoltaic system was developed to power mobile devices. Using the proposed scenario, an experiment was carried out in a school in the province of Salta, the results achieved were positive and highly significant from the educational point of view.
\end{abstract}

Palabras clave: Educación rural, dispositivos móviles, energía solar fotovoltaica

\section{Introducción}

Las zonas rurales aisladas de la Argentina se caracterizan, entre otros aspectos, por su baja densidad demográfica, cobertura de red celular limitada y carencia de servicio de distribución de energía eléctrica. Los habitantes de estas zonas utilizan energías alternativas, como paneles solares y grupos electrógenos, para cubrir necesidades energéticas elementales. La región Noroeste de Argentina (NOA) posee numerosas zonas de este tipo, donde las condiciones de vulnerabilidad económica, la baja densidad poblacional y/o el aislamiento geográfico (grandes distancias entre las viviendas y las 
escuelas o los centros urbanos), motivaron la creación de albergues dentro de las escuelas como una forma de garantizar que los chicos asistan cotidianamente a clases. En algunos casos, los alumnos viven en los albergues durante toda la semana (de lunes a viernes) y en otros, permanecen durante todo el ciclo lectivo o en períodos específicos.

Las escuelas albergue del NOA disponen de un plantel docente muy reducido, generalmente un director de establecimiento y un profesor que imparte educación en los diferentes grados. Cuentan con la asistencia de un profesor itinerante que recorre un itinerario de escuelas con el fin de orientar y apoyar al maestro en el desarrollo de sus tareas educativas, detectando y desarrollando las capacidades reales que cada niño posee. En algunas escuelas el profesor itinerante proporciona servicios directamente al alumno, coordinando la instrucción con el maestro.

En estos establecimientos educativos el aprendizaje mediado por tecnologías es prácticamente inexistente debido a las limitaciones energéticas de la zona y al elevado consumo de los equipos computacionales [Magadán, 2008]. Sin embargo, los dispositivos móviles (Celulares y Tablets) constituyen una alternativa viable para este tipo de escuelas, por su bajo consumo energético respecto de computadoras convencionales [Rocabado, 2015], haciendo posible que los alumnos se nutran de nuevas estrategias de aprendizaje que contribuyen a reducir la brecha digital existente entre los alumnos de los centros urbanos y rurales [Tedesco, 2015].

En este artículo se presenta una propuesta tecnológica que posibilita el acceso a Internet a docentes y alumnos de escuelas albergue, mediante el uso de dispositivos móviles de bajo consumo energético, utilizando energía solar fotovoltaica para la recarga de las baterías. Se presentan, además, los resultados cualitativos obtenidos en la implementación de nuestra propuesta en una escuela albergue, donde el maestro de la escuela desarrolló una clase de Historia con la colaboración de un profesor itinerante, quien brinda soporte para el uso de TDICs en el aula. La experiencia se realizó en el Paraje Potrero de Chañi, Departamento de Rosario de Lerma, Provincia de Salta, más precisamente en la Escuela Albergue “4422”. Dicho lugar se encuentra a 150 kilómetros de distancia de la ciudad de Salta (localidad capital de la provincia). En la Figura 1 se muestra una imagen del establecimiento y se lo ubica geográficamente, indicando el mismo con un círculo rojo.

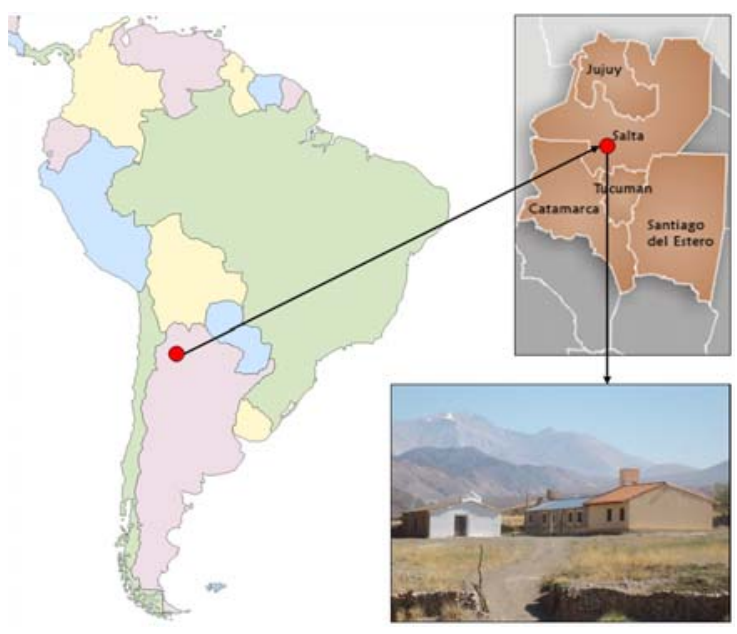

Figura 1. Ubicación geográfica del establecimiento educativo "4422". 
VI Congresso Brasileiro de Informática na Educação (CBIE 2017)

Anais do XXIII Workshop de Informática na Escola (WIE 2017)

\section{Recursos tecnológicos utilizados}

\subsection{Redes móviles ad-hoc}

Una red móvil ad-hoc o MANET (Mobile Ad hoc NETworks en inglés) [IETF MANET, 2010] es una colección de nodos inalámbricos móviles que se comunican de manera espontánea y autoorganizada constituyendo una red temporal sin la ayuda de ninguna infraestructura preestablecida (como puntos de acceso WiFi o torres de estaciones base celulares) ni administración centralizada. Los equipos o nodos que forman parte de ella (Celulares, Tablets y Netbooks), se organizan por si mismos para ayudarse los unos a los otros en el proceso de transportar paquetes de datos entre un origen y un destino. Por tanto las MANET dan un paso más en cuanto a movilidad (todos los nodos de la red pueden ser móviles) y flexibilidad (no se requiere inversión en infraestructura, y se minimiza la gestión de la red pues se auto-organiza ella misma)[Cordeiro de Morais, 2011].

Las MANET pueden ser redes autónomas o subordinadas si se encuentran conectadas o integradas a otras redes externas con infraestructura (Internet, redes corporativas extranets o intranets). En la Figura 2 se ilustra un ejemplo de MANET subordinada que se conecta a Internet utilizando los servicios de la red celular, esta configuración posibilita a los usuarios acceder a contenidos digitales desde dispositivos móviles que forman parte de la MANET. El despliegue de la MANET se realiza utilizando estándares de comunicaciones inalámbricas de corto alcance como Bluetooth [Bluetooth SIG, 2010] o WiFi [IEEE 802.11 Working Group, 2005], mientras que para la conexión a Internet se utiliza la red celular con tecnología 2G (GSM/GPRS), 3G (UMTS) o 4G (LTE).

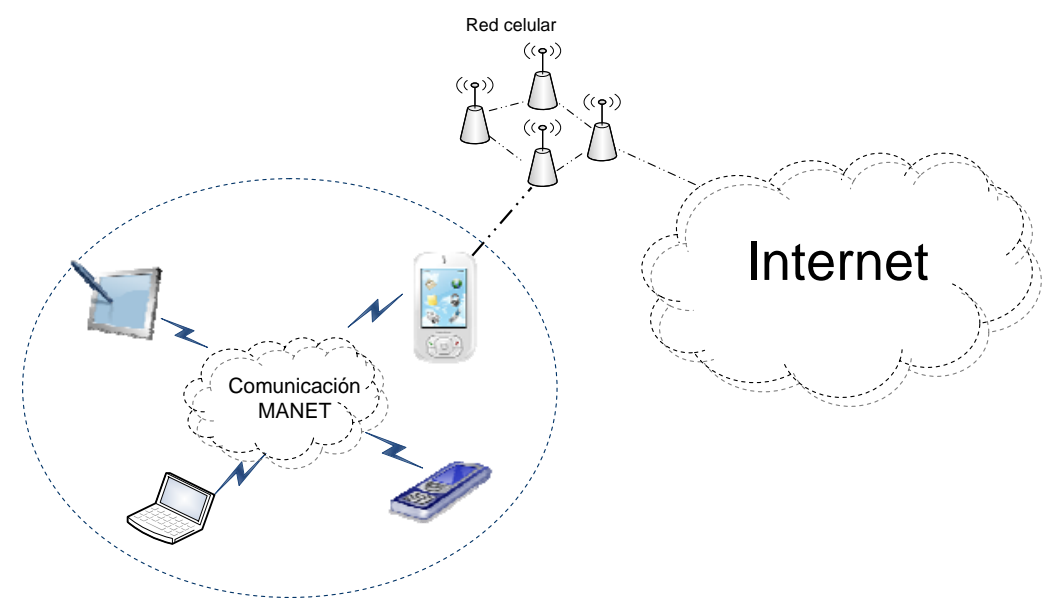

Figura 2. MANET subordinada a Internet.

Por sus características las MANETs constituyen una tecnología ideal para facilitar servicios de comunicación a dispositivos móviles en zonas aisladas donde no es posible montar y configurar redes de infraestructura debido a inconvenientes físicos y recursos limitados, como la energía y/o cobertura de red celular.

En trabajos previos [Rocabado y Herrera, 2013] y [Rocabado y Cadena, 2016] se propone el uso de MANETs para aplicar estrategias de m-learning en escuelas ubicadas en zonas rurales aisladas de la provincia de Santiago del Estero. En ambos trabajos se relatan experiencias exitosas donde un profesor de secundaria desarrolla su 
clase utilizando objetos de aprendizaje almacenados en un servidor de recursos mlearning, estos objetos fueron accedidos por los alumnos desde sus teléfonos celulares. Durante el desarrollo de estas experiencias se evidenció que el uso de recursos tecnológicos como Bluetooth para desplegar la MANET y GPRS para establecer la comunicación entre los nodos de la MANET y el servidor de contenidos, redujo de manera considerable el consumo de energía en los celulares sin afectar el rendimiento de las aplicaciones utilizadas para acceder a los contenidos m-learning.

\subsection{Consumo energético en dispositivos móviles}

En los últimos años se produjo una evolución tecnológica importante en los dispositivos móviles en general y en particular en los teléfonos celulares, los nuevos equipos llevan integrados una gran variedad de componentes para brindar a sus usuarios funcionalidades avanzadas. Lamentablemente, la velocidad a la que evolucionó la capacidad de las baterías no ha sido la misma; este inconveniente ha planteado que los fabricantes tengan que diseñar el hardware y el software de los dispositivos con premisas de eficiencia energética para reducir los niveles de energía consumidos por estos equipos. No obstante, si un dispositivo va a ser utilizado en zonas aisladas donde las posibilidades de recarga son limitadas, será necesario implementar mecanismos que permitan administrar y optimizar el uso de la energía disponible.

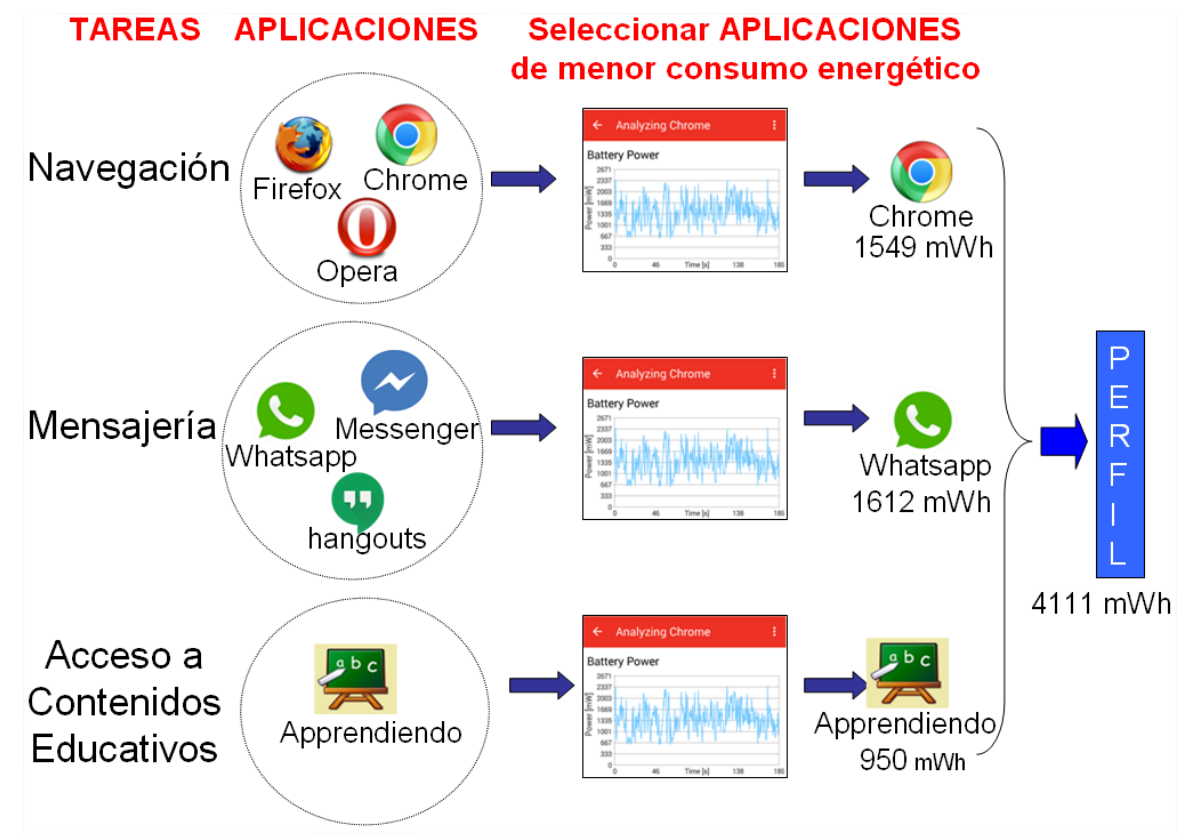

Figura 3. Perfiles de consumo energético

En este sentido, nuestro grupo de investigación desarrolló una propuesta metodológica [Rocabado Sergio y Cadena Carlos, 2015] que permite determinar los requerimientos energéticos de un celular para ejecutar tareas de uso frecuente en zonas rurales. Haciendo uso de la misma, se seleccionan las aplicaciones de menor consumo energético para cada tipo de tarea (eficiencia energética) y se definen perfiles de consumo de energía en función de los requerimientos informáticos de los pobladores y/o visitantes de la zona. A manera de ejemplo en la Figura 3 se ilustra un perfil de consumo que agrupa tres tareas: Navegación, Mensajería y Acceso a contenidos educativos. Se observa que las aplicaciones de menor consumo energético son: Chrome 
VI Congresso Brasileiro de Informática na Educação (CBIE 2017)

Anais do XXIII Workshop de Informática na Escola (WIE 2017)

para la navegación, Whatsapp para mensajería y Apprendiendo! para acceso a contenidos educativos. El total de consumo para el perfil es de $4111 \mathrm{mWh}$.

\subsection{Energía solar fotovoltaica}

El recurso energético renovable más utilizado en la región del NOA es la energía solar, ya que la aridez del clima y la latitud tropical hacen que se cuente con una alta radiación solar la mayoría de los días del año. Se trata de un recurso limpio y renovable que puede ser aprovechado en poblaciones rurales aisladas que se encuentran fuera del alcance de los centros de distribución de energía [Ottavianelli Emilce y Cadena Carlos, 2013].

La tecnología fotovoltaica actualmente ya es competitiva para proveer de energía eléctrica a lugares alejados del Sistema Interconectado Nacional (SIN). Entre muchas de sus ventajas pueden mencionarse las siguientes: Se evita el costoso tendido y mantenimiento de las líneas eléctricas en zonas de difícil acceso y de escasa población, una vez instalada tiene un costo energético nulo, posee bajo costo de mantenimiento y un riesgo de fallas muy bajo.

Si bien los sistemas fotovoltaicos actualmente son utilizados en muchas zonas aisladas, el aprovechamiento de la energía solar para la carga de dispositivos móviles no está lo suficientemente difundido. En este trabajo se introduce la aplicación de esta tecnología en escuelas albergue, contribuyendo a mejorar las posibilidades de comunicación de la comunidad educativa de las mismas.

Como parte de esta propuesta se presenta un cargador solar de tamaño y peso reducido que interactúa con el dispositivo móvil. Los datos de radiación solar y temperatura que entrega el cargador permiten estimar la energía disponible para el dispositivo. Esta energía se administra planificando la ejecución de aplicaciones en función de sus requerimientos energéticos.

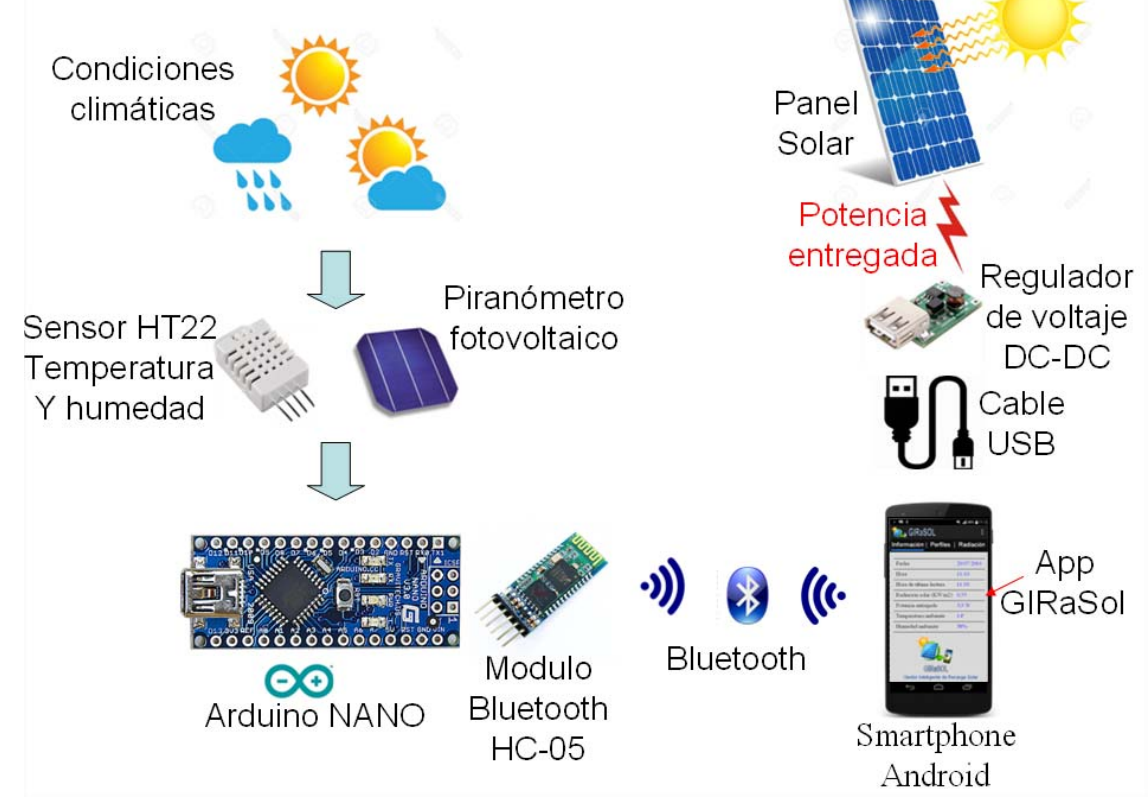

Figura 4. Prototipo de Sistema Fotovoltaico. 
VI Congresso Brasileiro de Informática na Educação (CBIE 2017)

Anais do XXIII Workshop de Informática na Escola (WIE 2017)

El prototipo (Figura 4) se construyó a partir de un panel fotovoltaico de tamaño reducido al cual se le agregaron sensores de radiación solar, temperatura y humedad y una placa de control (Arduino BT). Los sensores están conectados a la placa Arduino BT, en la cual se ejecuta un programa de control que periódicamente recolecta los valores de los sensores y los almacena en una memoria flash para ser enviados al dispositivo móvil cuando son requeridos.

Para comunicar el dispositivo móvil con el cargador se desarrolló la aplicación móvil GIRaSol (Gestión Inteligente de Recarga Solar), esta aplicación se comunica a través de Bluetooth con la placa Arduino instalada en el cargador, obtiene y utiliza los datos almacenados en la memoria flash para estimar la cantidad de energía que el panel solar puede suministrar al dispositivo móvil. Antes de utilizar GIRaSol se deben definir perfiles de aplicaciones y determinar el consumo energético de cada perfil utilizando la metodología presentada en el apartado anterior, los perfiles y su consumo energético se cargan en GIRaSol mediante un formulario de carga de datos. Conociendo la potencia que el panel puede entregar y el consumo energético de cada perfil, GIRaSol informa al usuario las franjas horarias en las que es posible ejecutar un perfil de aplicaciones.

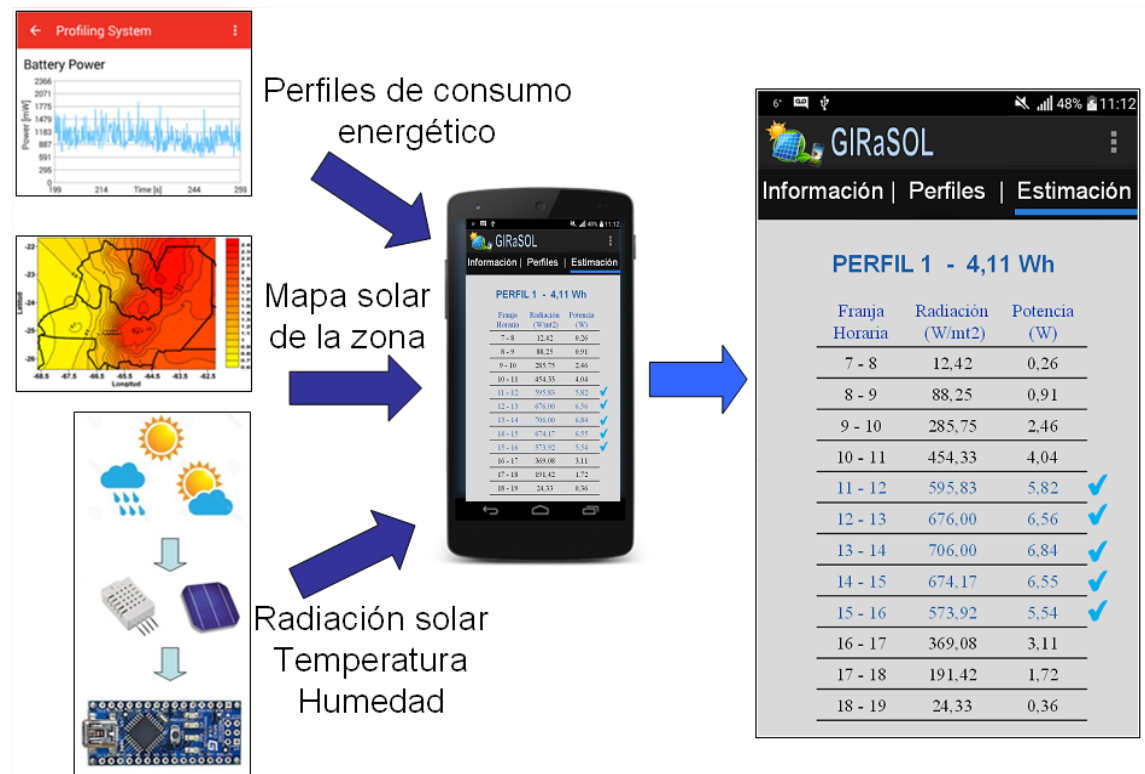

Figura 5. Aplicación móvil GIRaSol

En la Figura 5 se muestra una captura de pantalla con los resultados de GIRaSol para el perfil ejemplo de la Figura 1, se observa que la energía que el panel solar entrega alcanza para ejecutar las aplicaciones del perfil solamente de 11 a 16 horas.

\section{Escenario de trabajo}

En la figura 6 se presenta el escenario de trabajo utilizado para realizar la experiencia en la escuela albergue. La finalidad principal es la de proporcionar a los dispositivos móviles, acceso a contenido digital alojado en servidores Web de Internet, sin comprometer recursos como el ancho de banda y la energía que son limitados en la zona de despliegue. 


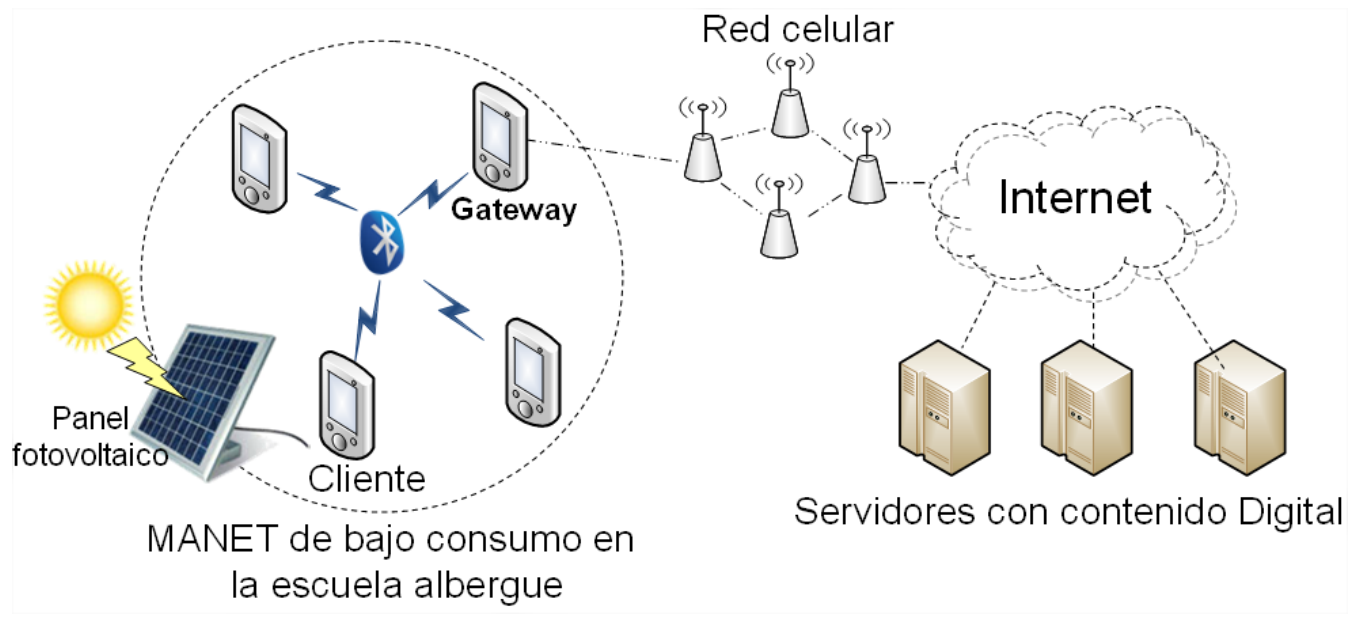

Figura 6. Escenario de trabajo.

Se realizó el despliegue de una MANET utilizando tecnología Bluetooth en modo PAN [JOHANSSON, 2001]con un nodo maestro y tres nodos esclavos. Para optimizar el uso del ancho de banda, el nodo maestro se conecta en forma directa a Internet utilizando los servicios de la red celular, mientras que los nodos esclavos lo hacen a través del nodo maestro que actúa como pasarela de comunicación (Gateway) entre la MANET y la red Celular.

Para asegurar el suministro de energía a los dispositivos móviles se utilizó el pico sistema fotovoltaico con placa de control Arduino (prototipo presentado en el apartado 2.3).

\section{Desarrollo de la experiencia}

El montaje de la experiencia estuvo a cargo de integrantes del equipo de investigación del cual forman parte los autores de este trabajo, utilizando el siguiente equipamiento de nuestro laboratorio: 4 celulares Samsung Galaxy J5 con chipset Qualcomm Snapdragon 410, 1 Sistema fotovoltaico portátil (prototipo de la Figura 4) y 3 paneles fotovoltaicos simples.

Se contó con la colaboración del docente itinerante y de la maestra de la escuela albergue, quienes fueron capacitados en los siguientes temas: Principios básicos de la energía solar fotovoltaica, sistemas fotovoltaicos portátiles, uso de la aplicación GIRaSol, consumo energético de aplicaciones móviles, configuración de una MANET Bluetooth y conexión de un dispositivo móvil a la red celular.

En primer lugar se realizó la configuración del escenario de trabajo, se procedió entonces a:

- Medir el consumo energético de la aplicación seleccionada (Chrome) para realizar las consultas en Internet.

- Cargar el perfil de consumo para Chrome en GiRaSol.

- Instalar el cargador solar portátil, direccionando el panel para optimizar la ganancia de radiación solar.

- Conectar el celular maestro a la placa Arduino del cargador solar y ejecutar la aplicación GiRaSol para determinar las bandas horarias en las cuales se puede ejecutar el perfil de consumo de la aplicación Chrome. 
VI Congresso Brasileiro de Informática na Educação (CBIE 2017)

Anais do XXIII Workshop de Informática na Escola (WIE 2017)

- Conectar los dispositivos esclavos a paneles fotovoltaicos simples.

- Configurar Bluetooth, el celular del profesor en modo maestro y los dispositivos de los alumnos en modo esclavo.

- Verificar el acceso a Internet desde el dispositivo maestro y desde los dispositivos esclavos.

A continuación se desarrolló una clase de Historia con la participaron de la maestra y 10 alumnos de ambos sexos cuyas edades variaban entre 11 y 14 años, durante la explicación de la profesora no estaba permitido el uso de celulares. Al finalizar la maestra organizó a los alumnos en 3 grupos y entregó un cuestionario y un celular a cada grupo. Bajo la supervisión del profesor itinerante, los alumnos de los 3 grupos respondieron a las preguntas del cuestionario, utilizando los celulares para realizar consultas en Internet.

\section{Resultados de la experiencia}

El despliegue de la MANET permitió a los alumnos acceder a los contenidos digitales de manera fácil, rápida y transparente, de tal forma que no fue necesario que los alumnos posean conocimientos de redes para manejar la aplicación elegida para navegar por Internet (Chrome).

El uso de la tecnología generó un ambiente de entusiasmo y colaboración, los alumnos trabajaron interactuando permanentemente entre sí y también con los profesores. Los 3 grupos respondieron el cuestionario en forma correcta y dentro de los límites de tiempo establecidos por los docentes.

Algunos alumnos tenían experiencia previa en el uso de aplicaciones móviles, ya que son propietarios de celulares. Estos alumnos manifestaron interés en el uso de sus dispositivos como recurso de aprendizaje dentro y fuera del aula.

Por otra parte, se realizaron entrevistas a la maestra, al profesor itinerante y a la directora del establecimiento educativo, quienes manifestaron que veían esta experiencia como algo novedoso que motiva a los alumnos y permite mejorar el aprendizaje.

\section{Conclusiones y trabajos futuros}

En las zonas rurales aisladas la instalación de redes eléctricas tiene un elevado costo debido a las distancias, a las dificultades de acceso y al escaso número de pobladores. El prototipo presentado apunta a solucionar esta carencia con una propuesta sustentable de bajo costo, haciendo uso de componentes económicos y energía renovable.

El uso de la energía solar fotovoltaica no solamente posibilita el uso de dispositivos móviles en escuelas que no tienen acceso a la energía eléctrica, sino que de forma indirecta introduce las energías renovables en la vida de estas comunidades educativas, pudiendo concientizar a docentes y alumnos sobre las ventajas del uso de una fuente de energía renovable.

Desde el punto de vista educativo, los resultados fueron satisfactorios en función del aprendizaje observado en los alumnos y de las entrevistas realizadas. Las principales contribuciones de la propuesta son: 
VI Congresso Brasileiro de Informática na Educação (CBIE 2017)

Anais do XXIII Workshop de Informática na Escola (WIE 2017)

- Disminución de la brecha digital existente entre establecimientos educativos rurales y establecimientos urbanos, posibilitando que alumnos de escuelas rurales accedan a contenidos digitales educativos alojados en servidores Web de Internet.

- Incremento de las posibilidades de comunicación de docentes y alumnos de escuelas albergue, permitiendo el acceso a aplicaciones de Internet tales como correo electrónico, mensajería y redes sociales.

- Mejoras en el uso de la tecnología disponible en zonas aisladas, teniendo en cuenta que algunos alumnos de estas escuelas son propietarios de equipos celulares que utilizan como reproductores de música o cámaras fotográficas y no como dispositivos de comunicación.

Para finalizar y con base en los resultados obtenidos en la experiencia, se puede concluir que "el uso de dispositivos móviles abastecidos con energía solar fotovoltaica se constituye como una alternativa tecnológica que introduce mejoras en el proceso de enseñanza aprendizaje en comunidades escolares rurales aisladas, posibilitando a los docentes el empleo de TDICs y acercando las tecnologías de la información y comunicación a niños de estas comunidades”

\section{Referencias}

Bluetooth SIG, Special Interest Group. (2010). Bluetooth v4.0 low energy (LE). from http://www.bluetooth.com/Pages/Low-Energy.aspx

Cordeiro de Morais, Carlos and Agrawall Dharma. (2011). Wireless PANs. In W. S. Publishing (Ed.), Ad Hoc and Sensor Networks - Theory and Applications (pp. 196-258). Singapore: World Scientific Publishing.

IEEE 802.11 Working Group. (2005). IEEE Standard for Wireless Local Area Networks. IEEE, New York, USA.

IETF MANET. (2010). MANET Active Work Group. http://tools.ietf.org/wg/manet

JOHANSSON, Per. (2001). Bluetooth - an Enabler for Personal Area Networking. IEEE Network (Ericsson Research).

Magadán, C. (2008). Las Tic en Escuelas rurales de La Argentina. Las TIC: del aula a la agenda política, Ponencias del Seminario internacional Cómo las TIC transforman las escuelas. IIPE-UNESCO, Sede Regional Buenos Aires, UNICEF.

Ottavianelli Emilce y Cadena Carlos. (2013). Uso de indicadores sociales en estudios de factibilidad de instalación de sistemas solares para generación de electricidad en localidades rurales. Paper presented at the XX Simposio peruano de Energía solar, Tacna - Perú.

Rocabado, Sergio. (2015). Uso de dispositivos móviles inteligentes en zonas rurales aisladas abastecidas con energía solar fotovoltaica. Paper presented at the CACIC 2015.

Rocabado Sergio y Cadena Carlos. (2015). Cargadores solares portátiles para el uso de dispositivos móviles en zonas rurales aisladas del NOA. Paper presented at the ASADES 2015. 
VI Congresso Brasileiro de Informática na Educação (CBIE 2017)

Anais do XXIII Workshop de Informática na Escola (WIE 2017)

Rocabado Sergio y Cadena Carlos. (2016). M-learning en escuelas rurales. Paper presented at the TE\&ET 2016, Morón - Provincia de Buenos Aires - Argentina. ISBN: 978-987-3977-30-5.

Rocabado Sergio y Herrera Susana. (2013). M-learning en zonas de recursos limitados. Paper presented at the TE\&ET 2013, Santiago del Estero - Argentina.

Tedesco, Steinberg y Tófalo. (2015). Principales resultados de la Encuesta Nacional sobre Integración de TIC en la Educación Básica Argentina. Programa TIC y Educación Básica. Informe general. Fondo de las Naciones Unidas para la Infancia (UNICEF). 